\title{
Linear Solution Scheme for Microstructure Design with Process Constraints
}

\author{
Pinar Acar* and Veera Sundararaghavan $\ddagger$ \\ University of Michigan, Ann Arbor, Michigan 48109
}

DOI: $\underline{10.2514 / 1 . J 055247}$

\begin{abstract}
This paper addresses a two-step linear solution scheme to find an optimum metallic microstructure satisfying performance needs and manufacturability constraints. The microstructure is quantified using the orientation distribution function, which determines the volume densities of crystals that make up the polycrystal microstructure. The orientation distribution function of polycrystalline alloys is represented in a discrete form using finite elements, and the volume-averaged properties are computed. The first step of the solution approach identifies the orientation distribution functions that lead to the set of optimal engineering properties using linear programming. This step leads to multiple solutions, of which only a few can be manufactured using traditional processing routes such as rolling and forging. In the second step, textures from a given process are represented in a space of reduced basis coefficients called the process plane. This step involves generation of orthogonal basis functions for representing spatial variations of the orientation distribution functions during a given process using proper orthogonal decomposition. Multiple orientation distribution function solutions in step one are then projected onto these basis functions to identify which of the optimal textures are feasible through a given manufacturing process. This feasibility is determined with two approaches. The first approach finds the closest match to the orientation distribution function solutions in the material plane, whereas the second approach finds the closest match to a desired set of properties instead of the orientation distribution functions. The method is explained through an example of vibration tuning of a galfenol alloy, with the primary objective of maximizing the yield strength.
\end{abstract}

\section{Nomenclature}

$A=$ orientation distribution function

$A_{1} \quad=$ one orientation distribution function solution for linear solver

$a=$ reduced-order model coefficients

$C \quad=$ spatial correlation matrix

$C^{\text {eff }}=$ effective stiffness tensor

$\boldsymbol{d}=$ vector of desired material properties

$E_{1} \quad=$ Young's modulus along axis-1

$\boldsymbol{e}=$ error vector for desired property matching

$G_{12}=$ shear modulus in 1-2 plane

$I_{p}=$ polar inertia moment

$I_{1} \quad=$ moment of inertia along axis-1

$J=$ torsion constant

$L \quad=$ beam length

$L=$ macrovelocity gradient

$m \quad=$ unit mass

$N=$ number of independent nodes

$\mathcal{N}=$ number of snapshots

$q=$ volume fraction

$r=$ orientation

$r \quad=\quad$ error vector for orientation distribution function matching

$S=$ compliance

$t \quad=$ time

$\boldsymbol{V}=$ null-space vector

$\epsilon=$ volume-averaged strain

$\rho \quad=$ density

Received 1 April 2016; revision received 23 May 2016; accepted for publication 28 May 2016; published online 17 August 2016. Copyright (C) 2016 by Pinar Acar. Published by the American Institute of Aeronautics and Astronautics, Inc., with permission. Copies of this paper may be made for personal and internal use, on condition that the copier pay the per-copy fee to the Copyright Clearance Center (CCC). All requests for copying and permission to reprint should be submitted to $\mathrm{CCC}$ at www.copyright.com; employ the ISSN 0001-1452 (print) or 1533-385X (online) to initiate your request.

*Graduate Research Assistant, Department of Aerospace Engineering. Student Member AIAA.

${ }^{\dagger}$ Associate Professor, Department of Aerospace Engineering. Member AIAA.

$$
\begin{array}{ll}
\sigma & =\text { volume-averaged stress } \\
\sigma_{y} & =\text { yield stress } \\
\phi & =\text { basis function } \\
\chi & =\text { orientation dependent property } \\
\omega_{1 b} & =\text { first bending natural frequency } \\
\omega_{1 t} & =\text { first torsion natural frequency }
\end{array}
$$

\section{Introduction}

$\mathbf{R}$ EALIZATION of optimal material properties is important for hardware components in aerospace applications where there is a continuous need to reduce material utilization for reduced process cost, fuel consumption, and higher mobility. Critical components involve performance indices that are directly related to microstructures obtained during processing. This calls for direct control of microstructure evolution using well-designed processes. Property cross plots, which are a standard approach for materials selection, as generalized by Ashby [1], have allowed graphical quantification of property-performance relations. Recent developments in materials by design have allowed a more advanced systems approach that integrates processing, structure, and property through multiscale computational material models [2]. In the area of composites, techniques that enable tailoring of microstructure topology have allowed the design of structures with interesting extremal properties such as negative thermal expansion [3] and a negative Poisson's ratio [4]. In contrast to composites, techniques that allow tailoring of properties of polycrystalline alloys involve tailoring of preferred orientation of crystals manifested as the crystallographic texture. During forming processes, mechanisms such as crystallographic slip and lattice rotation drive the formation of texture and variability in property distributions in such materials. A useful method for designing materials is through control of deformation processes leading to the formation of textures that yield desired property distributions.

The microstructure modeling of the present work is based on the quantification of the microstructure using the orientation distribution function (ODF). The ODF represents the volume fractions of the crystals of different orientations in the microstructure. The ODF is defined based on a parameterization of the crystal lattice rotation. Popular representations include Euler angles [5,6] and classes of angle-axis representations, with the most popular being the Rodrigues parameterization [7]. Conversion of continuous 
orientation space to finite degrees of freedom for material property optimization requires discretization techniques. Discretization schemes either focus on a global basis (e.g., Fourier space or spherical harmonics [ $[$, 9$]$ ]) or a local basis using a finite element discretized Rodrigues space with polynomial shape functions defined locally over each element $[10,11]$. Step one of the microstructure optimization problem involves computation of the discretized ODF values that satisfy a given set of desired properties. Liu et al. [12] achieved this by directly sampling the ODF space using a datamining methodology. However, the space of all possible ODFs is high-dimensional and sampling in the property space is favorable because the number of design variables is significantly lesser. Adams et al. [8], Kalidindi et al. [9], and Fast et al. [13] employed sampling within the property hull similar to this work, but they employed a Fourier basis for discretizing the ODF. In a previous paper [14], we demonstrated the use of the finite element representation of the ODF to compute optimal ODF solutions to an engineering problem. The approach presented in that work led to multiple ODF solutions.

The motivation of the present work is to find out which of the multiple ODFs identified from this optimization step can be manufactured using a set of deformation processes. Li et al. [15] addressed this problem by representing processing paths as streamline functions in the space of spectral coefficients. This allowed inversion of processing paths by tracking streamlines connecting the initial and optimal textures. The complexity of the model depended on the number of spectral coefficients used to represent texture. Since a large number of spectral terms were needed to capture sharp textural features, the complexity of the models used to describe processing paths increased accordingly. In this work, textures from a given process are represented by using basis functions that are derived using proper orthogonal decomposition (POD) [16]. The multiple optimum ODFs from step one are projected onto the basis functions of various deformation processes, and the optimal process is identified as the one that minimizes the distance between any one of the optimal ODFs and the ODF that can be achieved from a process. A second approach where the optimal property values are projected into the process basis is also presented. The remainder of this paper is organized as follows. Section II addresses the computation of microstructure properties and reduced-order models of the ODF. In Sec. III, we discuss the features of the galfenol beam vibration tuning problem and the optimization results. Section IV includes identification of the optimal processes using linear solution methodologies. A summary of the paper and potential extensions are finally discussed in Sec. $\underline{\text { V. }}$

\section{Modeling Properties of Microstructures}

The alloy microstructure consists of multiple crystals, with each crystal having an orientation. The generalized Hooke's law for the aggregate of crystals may be written in the form

$$
<\sigma_{i j}>=C_{i j k l}^{\mathrm{eff}}<\epsilon_{k l}>
$$

where $<\epsilon_{k l}>$ and $<\sigma_{i j}>$ are the volume-averaged strain and stress, respectively, in the aggregate; and $C^{\text {eff }}$ is the effective stiffness tensor in sample coordinate system. Assuming homogeneity of the deformation in a macroscale elementary volume, the effective elastic properties may be found through averaging using the Taylor approximation [17]:

$$
C^{\mathrm{eff}}=<C>
$$

where $C$ is the stiffness tensor for each crystal in the sample coordinate frame and the average is done over the aggregate of crystals. If the effect of factors (e.g., crystal size and shape) is ignored, averaging (denoted by $\langle\cdot\rangle$ in the preceding equation) can be performed over the orientation distribution function (represented by $A$ ). The ODF gives the volume density of each orientation in the microstructure. If the orientation-dependent property for single crystals $\chi(r)$ is known, any polycrystal property can be expressed as an expected value, or average, in the orientation space given by

$$
<\chi>=\int_{R} \chi(r) A(r, t) \mathrm{d} v
$$

where the ODF $(A \geq 0)$ is a function of orientation $r$ and time $t$ (during processing), and it satisfies the normalization constraint

$$
\int_{R} A(r, t) \mathrm{d} v=1
$$

\section{A. Property Representation in Rodrigues Space}

The present work employs the axis-angle parameterization of the orientation space proposed by Rodrigues as discussed in Kumar and Dawson [10]. Angle-axis representations define an alternate way of representing orientations compared to Euler angles $[5,18]$. The Rodrigues parameterization is created by scaling the axis of rotation $n$ as $r=n \tan (\theta / 2)$, where $\theta$ is the rotation angle. Finite element discretization of the orientation space (as shown in Fig. 1) and associated integration schemes using Gauss quadrature allow a matrix representation of Eq. (3). The ODF is discretized into $N$ independent nodes with $N_{\text {elem }}$ finite elements and $N_{\text {int }}$ integration points per element. Using this parametrization, any polycrystal property can be expressed in a linear form as follows [16]:

$$
\begin{aligned}
<\chi> & =\int_{R} \chi(r) A(r, t) \mathrm{d} v \\
& =\sum_{n=1}^{N_{\text {elem }}} \sum_{m=1}^{N_{\text {int }}} \int_{R} \chi\left(r_{m}\right) A\left(r_{m}\right) w_{m}\left|J_{n}\right| \frac{1}{\left(1+r_{m} \cdot r_{m}\right)^{2}}
\end{aligned}
$$

where $A\left(r_{m}\right)$ is the value of the ODF at the $m$ th integration point with global coordinate $r_{m}$ of the $n$th element, $\left|J_{n}\right|$ is the Jacobian determinant of the $n$th element, $w_{m}$ is the integration weight associated with the $m$ th integration point, and

$$
\frac{1}{\left(1+r_{m} \cdot r_{m}\right)^{2}}
$$

represents the metric of Rodrigues parameterization. This is equivalent to an equation linear in the ODF: $\langle\chi\rangle=p^{\text {int }^{T}} A^{\text {int }}$, where

$$
p_{l}^{\text {int }}=\chi\left(r_{i}\right) w_{i}\left|J_{i}\right| \frac{1}{\left(1+r_{i} \cdot r_{i}\right)^{2}}
$$

and $A^{\text {int }}=A\left(r_{i}\right), \quad i=1, \ldots, N_{\text {int }} \times N_{\text {elem }}$. Using a smoothing operation, the equation can be written in terms of the independent nodes of the ODF (considering the crystallographic symmetry) as $<\chi>=p^{T} \boldsymbol{A}$. Similarly, the normalization constraint

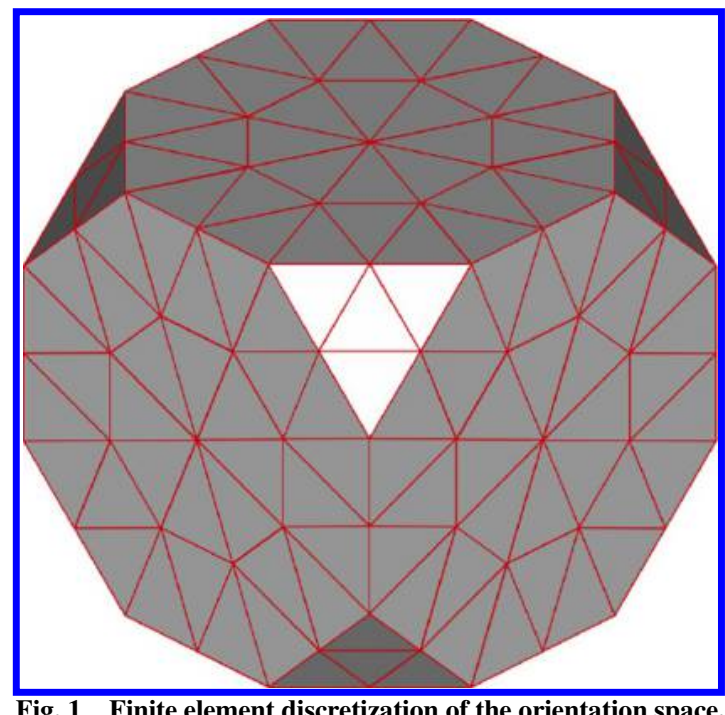




$$
\int_{R} A(r, t) \mathrm{d} v=1
$$

leads to a linear equation $\boldsymbol{q}^{T} \boldsymbol{A}=1$. The space of all possible ODFs is in the form of a hyperplane satisfying the normalization $\left(\boldsymbol{q}^{T} \boldsymbol{A}=1\right)$ and positiveness $(\boldsymbol{A} \geq 0)$ constraints and is called the "material plane."

The polycrystal stiffness $\overline{\boldsymbol{C}}$ is computed through a weighted average (over $\boldsymbol{A}$ ) of the stiffnesses of individual crystals expressed in the sample reference frame. In a general case, use of Eq. (2) to compute the effective stiffness tensor results in an anisotropic stiffness tensor. The yield stress is computed using a crystal plasticity model from recent work [12].

\section{B. Reduced-Order Model of the ODF}

The discussion here follows the work in [19-21], where model reduction of crystal plasticity was first introduced using the technique of POD. Model reduction involves generation of basis functions optimal for representing ODFs obtained from a process path. Using such basis functions, any ODF $[\boldsymbol{A}(\boldsymbol{r}, \boldsymbol{t})]$ from the time history of ODF evolution in a given process can be approximated as follows:

$$
\mathcal{A}(\boldsymbol{r}, \boldsymbol{t})=\sum_{m=1}^{b} \boldsymbol{a}_{m}(\boldsymbol{t}) \boldsymbol{\phi}_{m}(\boldsymbol{r})
$$

In the preceding equation, $\boldsymbol{\phi}_{m}$ represents " $b$ " basis functions (independent of time) and $\boldsymbol{a}_{m}(\boldsymbol{t})$ denotes the corresponding time-dependent coefficients. Once such basis functions $\boldsymbol{\phi}_{m}$ are computed, time-dependent coefficients can be used to reconstruct the textures arising from the process path. Readers are referred to [19], where texture evolution is computed by using Eq. (5). Texture evolution can also be computed across a set of extrapolatory regimes of the process (i.e., conditions deviating from those used to generate the basis functions) using the same set of basis functions.

The "method of snapshots" is an efficient technique of obtaining basis functions from an ensemble of ODF data $\left[\mathcal{A}(\boldsymbol{r}, \boldsymbol{t})_{i=1}^{\mathcal{N}}\right]$ consisting of ODFs at various times during texture evolution over a deformation path. Here, the basis functions $\phi$ take the form [19]

$$
\boldsymbol{\phi}_{m}=\sum_{i=1}^{N} \boldsymbol{u}_{i}^{m} \mathcal{A}^{i}
$$

where $\mathcal{A}^{i}$ represent textures from the ensemble, and $\boldsymbol{u}_{i}^{m}$ is determined by solving the following linear eigenvalue problem:

$$
\boldsymbol{C U}=\boldsymbol{\Lambda} \boldsymbol{U}
$$

where $\boldsymbol{C}$ is the spatial correlation matrix defined as

$$
C_{i j}=\frac{1}{\mathcal{N}} \int_{\mathcal{R}} \mathcal{A}^{i}(r) \mathcal{A}^{j}(r) \mathrm{d} \boldsymbol{v}
$$

$\boldsymbol{\Lambda}$ and $\boldsymbol{U}$ comprise the eigenvalues and the eigenvectors of the system, respectively. To determine a suitable basis size $\boldsymbol{b}$, one must ensure that the eigenmodes selected capture as much "system energy" as possible. This is possible by selecting the basis functions that correspond to the largest eigenvalues in $\boldsymbol{\Lambda}$. Once the modes have been evaluated, the optimal basis is generated from Eq. (6). The coefficients $\boldsymbol{a}$ corresponding to any ODF in a deformation path can be retrieved from

$$
\boldsymbol{a}_{m}=\int_{\mathcal{R}} \mathcal{A}(r) \boldsymbol{\phi}_{m} \mathrm{~d} \boldsymbol{v}
$$

The space of reduced coefficients is called the process plane and satisfies the normalization and positiveness constraints of the ODF. The ODFs in a deformation path follow a curve in the space of reduced coefficients $a$. The success of the technique for representing texture evolution was shown in $[19,21,22]$, where just three basis functions were found to be sufficient for capturing most features of the evolving $\mathrm{ODF}$ in any given process. Basis functions are obtained for different processing modes using a 448-element discretization of the fundamental region. The basis used in the examples consists of modes generated from an ensemble of data obtained for tension, compression and shear processes up to time of $0.1 \mathrm{~s}$ when deformed with a strain rate of $1 \mathrm{~s}^{-1}$ using a time step of $\Delta t=0.01 \mathrm{~s}$. The basis depends upon the initial texture $\mathcal{A}(\boldsymbol{r}, \boldsymbol{t}=0)$ that is used in the solution of ODF evolution. However, the strength of POD analysis used here lies in the fact that the reduced basis works in extrapolatory modes to represent texturing under various deviations in the initial texture. As a result, ODFs resulting from processing to a different strain or processing a starting texture that deviates from the one used to build the basis are well approximated using the same set of basis functions. Different basis functions are generated to simulate different process sequences [22]. The reduced models used to represent the deformation paths are different, for example, when modeling the tension process on a rolled specimen compared to a process of tension acting on an annealed specimen with random texture. The basis functions for the tension, plane-strain compression, and shear processes are shown in Fig. $\underline{2}$. The process planes for these processes are shown in Fig. $\underline{3}$, by property (yield stress of galfenol).

\section{Range of Properties Obtainable from the Material Plane}

Property closures represent a complete range of properties obtainable from the space of ODFs. These are approximated by the space between upper and lower bounds of the given property. The upper-bound closure of stiffness values represents the range of properties obtainable by the upper-bound homogenization relation in Eq. (10). The lower-bound approach, which is based on a constantstress assumption, computes the properties in $\boldsymbol{C}^{-1}$ space. $\langle\boldsymbol{C}\rangle$ computations with upper- and lower-bound approaches are given in Eqs. (10) and (11), respectively:

$$
\begin{gathered}
<C>=\int_{R} C A \mathrm{~d} v \\
<C^{-1}>=\int_{R} C^{-1} A \mathrm{~d} v
\end{gathered}
$$

In both cases, the extremal ODFs correspond to the single crystals. The methodologies for developing the space of all possible properties (called property closure) are presented in [14]. The optimization is performed by sampling in the property space to identify the best combination of properties for a given engineering problem. A direct linear solver then determines the ODFs corresponding to the optimum properties in $C$ and $C^{-1}$ spaces. The solver is also capable of finding multiple/infinite solutions using the null space $\boldsymbol{V}_{i}$ of the coefficient matrix of the optimization problem. The methodology is explained in detail in another paper [14]. The infinite solutions can be represented as shown in the following:

$$
\boldsymbol{A}_{i}=\boldsymbol{A}_{1}+\lambda \boldsymbol{V}_{i}, \quad \text { where } i=1,2,3,4, \ldots, \boldsymbol{n}
$$

where Eq. (12) defines the infinite solutions $\boldsymbol{A}_{i}$, using one solution $\boldsymbol{A}_{1}$, and null-space vectors $\boldsymbol{V}_{i}$; and $\boldsymbol{n}$ is the number of null-space vectors. Even though the number of null-space vectors is finite, the number of solutions can be infinite because $\lambda$ can be any number that satisfies the ODF positiveness and normalization constraints.

\section{Vibration Tuning of a Cantilever Galfenol Beam}

The microstructure design involving vibration tuning of a cantilevered galfenol beam with a yield strength objective (Fig. 4) is explained here. The stiffness parameters and yield stress of the galfenol beam are represented using independent ODF values. The 


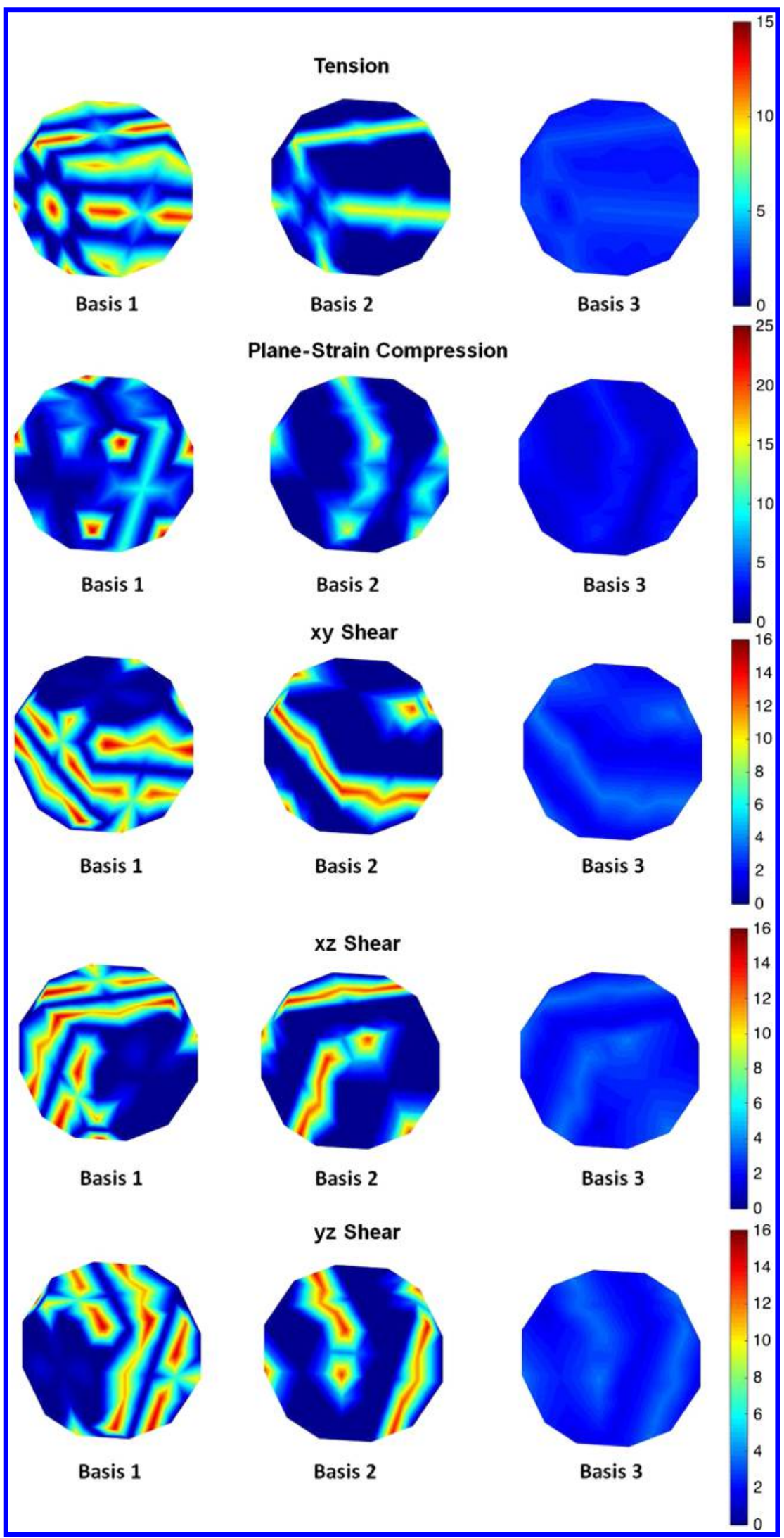

Fig. 2 Basis functions of different processes.

number of independent ODF values in this problem is 76 for the mesh considered here (Fig. 1). The goal of the problem is to find the ODFs that maximize yield stress while the first bending and torsional natural frequencies are constrained for vibration tuning.
According to the coordinate system introduced in Fig. 4, the analytical equations of the first torsional and bending natural frequencies for an orthotropic material can be shown, respectively, as follows: 


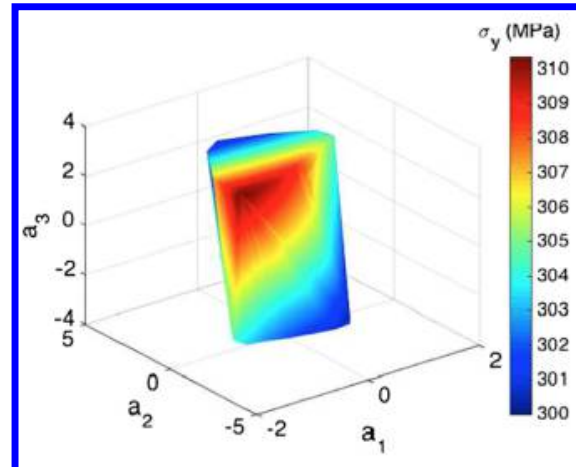

Tension Process Plane

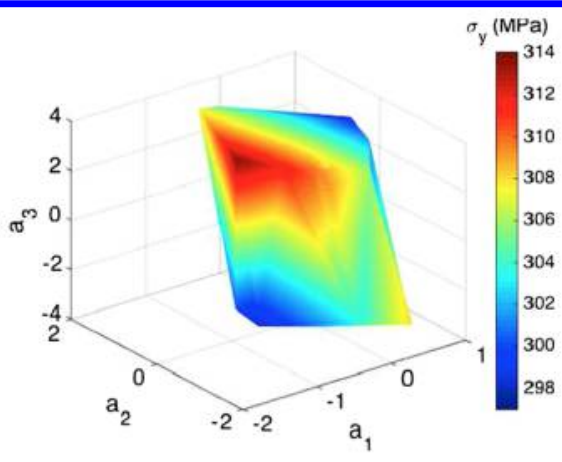

Compression Process Plane

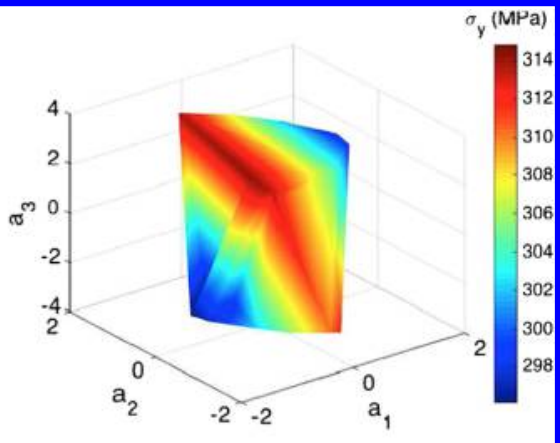

xy-Shear Process Plane

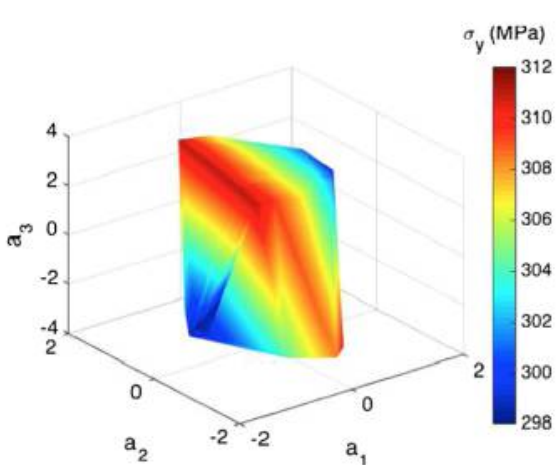

xz-Shear Process Plane

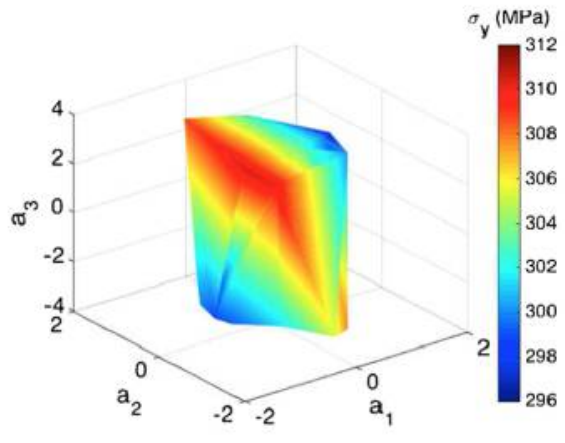

yz-Shear Process Plane

Fig. 3 Property closures for different deformation processes.

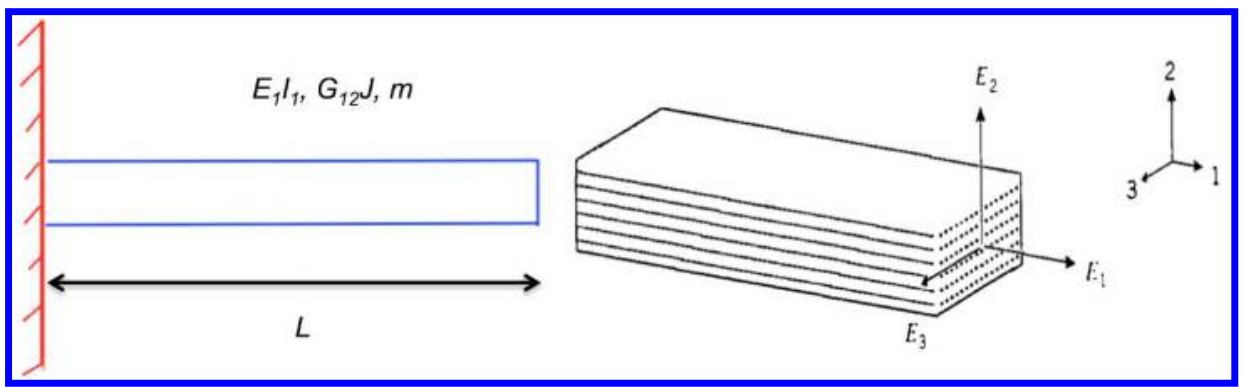

Fig. 4 Geometric representation of galfenol beam vibration problem.

$$
\begin{gathered}
\omega_{1 t}=\frac{\pi}{2 L} \sqrt{\frac{G_{12} J}{\rho I_{p}}} \\
\omega_{1 b}=(\alpha L)^{2} \sqrt{\frac{E_{1} I_{1}}{m L^{4}}} \text { and } \alpha L=1.87510
\end{gathered}
$$

where $G_{12}=1 / S_{66}, E_{1}=1 / S_{11}$, and $S$ are the compliance elements ( $\left.S=C^{-1}\right) ; E_{1}$ is the Young's modulus along axis 1 ; and $G_{12}$ is the shear modulus in the 1-2 plane. In these formulations, $J$ is the torsion constant, $\rho$ is density, $I_{p}$ is the polar inertia moment, $m$ is the unit mass, $L$ is the length of the beam, and $I_{1}$ is the moment of inertia along axis 1 . The computation of the yield stress using upper- and lower-bound approaches is given in Eqs. (15) and (16), respectively, in terms of single crystal yield strengths along the beam axis:

$$
\begin{aligned}
<\sigma_{y}> & =\int \sigma_{y} A \mathrm{~d} V \\
<\sigma_{y}^{-1}> & =\int \sigma_{y}^{-1} A \mathrm{~d} V
\end{aligned}
$$

The mathematical formulation of the optimization problem is given as follows:

$\max \sigma_{y}$

$$
\text { subject to } \int A \mathrm{~d} V=1
$$

$$
\text { subject to } 21.5 \mathrm{~Hz} \leq \omega_{1 t} \leq 23.5 \mathrm{~Hz}
$$

$$
\text { subject to } 100 \mathrm{~Hz} \leq \omega_{1 b} \leq 114 \mathrm{~Hz}
$$

$$
A \geq 0
$$

The optimization problem includes the normalization constraint as well as the constraints for the first natural frequencies to tune the 
beam vibration. To solve the problem, the length of the beam is taken as $L=0.45 \mathrm{~m}$ and the beam is considered to have a rectangular cross section with dimensions of $a=20 \mathrm{~mm}$ and $b=3 \mathrm{~mm}$. The steps taken to optimize the microstructure are summarized in the recent work of the authors [14].

\section{Results}

The multiple solutions of this problem correspond to the designs having the same values for microstructure dependent input parameters $\left(E_{1}\right.$ and $\left.G_{12}\right)$. The problem has 73 solution directions (76 optimization variables and three linear equations: two of them for computation of $E_{1}$ and $G_{12}$, and one of them for the unit volume constraint), and these solutions are polycrystal designs. The property closure graph for $E_{1}$ and $G_{12}$ variables $(E-G$ space $)$ is given in Fig. 5. The parameters of the multiple optimum solution are given in Table 1 . Some of the optimum microstructure designs are shown in Fig. 6. Since the linear solver was able to compute independent solution directions for galfenol beam optimization problem, each design in Fig. 6 is different than the others and has different ODF values. However, they still lead to an identical maximum yield stress value and satisfy the design constraints.

\section{A. Identification of ODFs Closest to an Optimal ODF in the Material Plane}

Five different processes were considered in this work. The ODFs from these processes are obtained using a specific macrovelocity gradient $L$ in the crystal plasticity solver [22] corresponding to these deformation processes. For example, the $x$ axis tension process is based on the following velocity gradient [22]:

$$
\boldsymbol{L}=\alpha_{1}\left[\begin{array}{ccc}
1 & 0 & 0 \\
0 & -0.5 & 0 \\
0 & 0 & -0.5
\end{array}\right]
$$

The location of optimal ODFs on the material plane does not convey information on how to realize such ODFs in practice. There may be several processing solutions to this problem. Here, we choose a particular processing path and check if it can closely produce one of the optimal textures. The optimal ODF from the material plane is assumed to be given by a perturbation $\boldsymbol{r}$ to an ODF in the process plane. We wish to minimize the perturbation in some sense, such that an ODF from the process plane is as close as possible to the optimal ODF in the material plane. In this work, the optimal ODFs on the material plane are the optimal ODF values found for galfenol vibration tuning problem.

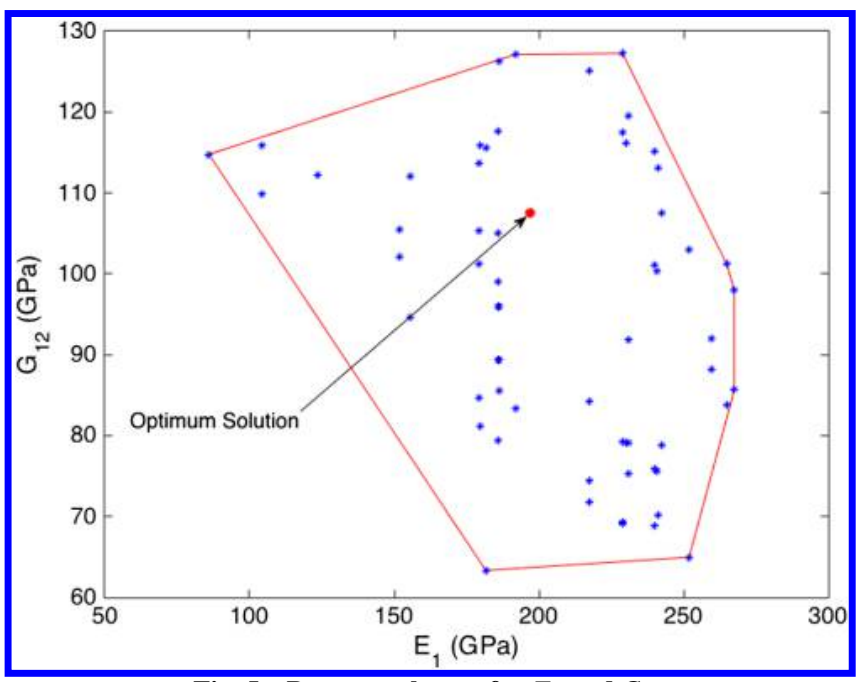

Fig. 5 Property closure for $E_{1}$ and $G_{12}$.
Table 1 Optimization results for vibration tuning of the galfenol beam

\begin{tabular}{lc}
\hline \hline Parameters & Optimum polycrystal design \\
\hline$\sigma_{y}$ & $308.4456 \mathrm{MPa}$ \\
$E_{1}$ & $188.8229 \mathrm{GPa}$ \\
$G_{12}$ & $93.6282 \mathrm{GPa}$ \\
\hline \hline
\end{tabular}

The optimal ODF in the process plane is written as

$$
\boldsymbol{A}_{\mathrm{opt}}=\sum_{m=1}^{b} \boldsymbol{a}_{m} \boldsymbol{\phi}_{m}+\boldsymbol{r}>0
$$

where

$$
\boldsymbol{A}=\sum_{m=1}^{b} \boldsymbol{a}_{m} \boldsymbol{\phi}_{m}
$$

provides the closest solution in the basis $\phi$, and $\boldsymbol{r}$ is the perturbation (or error) between the optimal ODF in the material plane $\left(\boldsymbol{A}_{i}=\boldsymbol{A}_{1}+\lambda_{i} \boldsymbol{V}_{i}\right)$ and the optimal ODF in the process plane. The normalization constraint is given as $\boldsymbol{q}^{T} \boldsymbol{A}_{i}=1$, and $\boldsymbol{q}^{T} \boldsymbol{A}=1$. The bound in the value of the solution ODF from the given ODF $\left(\boldsymbol{r}_{0} \geq 0\right)$ is minimized. The problem is posed as

$$
\min _{a} \boldsymbol{r}_{0}
$$

such that

$$
\sum_{m=1}^{b} \boldsymbol{a}_{m} \boldsymbol{\phi}_{m}+\boldsymbol{r}-\sum_{i=1}^{n} \lambda_{i} \boldsymbol{V}_{i}=\boldsymbol{A}_{1}
$$

The positivity of the optimal ODF dictates the constraints $\boldsymbol{A} \geq 0$ and $\boldsymbol{A}_{i} \geq 0$. In the solution procedure, the basis $\boldsymbol{\phi}_{m}$ and the error $\boldsymbol{r}$ are represented as a vector containing values at independent nodes (set of nodes representing distinct orientations while accounting for crystal symmetries). The ODFs in the process and material planes also require the positivity constraints

$$
\sum_{m=1}^{b} \boldsymbol{a}_{m} \boldsymbol{\phi}_{m} \geq 0
$$

and

$$
\boldsymbol{A}_{1}+\sum_{i=1}^{n} \lambda_{i} \boldsymbol{V}_{i} \geq 0
$$

The additional constraint on the bound in the value of the solution ODF from the given ODF is defined as $\left|\boldsymbol{r}_{i}\right| \leq \boldsymbol{r}_{0}$, which is equivalent to pairs of linear inequalities: $-\boldsymbol{r}_{i}-\boldsymbol{r}_{0} \leq 0$ and $\boldsymbol{r}_{i}-\boldsymbol{r}_{0} \leq 0$, $\boldsymbol{i}=1, \ldots, N$, where $\boldsymbol{N}$ is the number of independent nodes. This problem uses $\boldsymbol{x}=\left[\boldsymbol{r}_{1}, \ldots, \boldsymbol{r}_{N}, \lambda_{1}, \ldots, \lambda_{n}, \boldsymbol{a}_{1}, \ldots, \boldsymbol{a}_{b}, \boldsymbol{r}_{0}\right]^{T}$ as the variables to be identified. The error $\boldsymbol{r}_{i}$ from the nodal values of the optimal ODF is allowed to be of either sign. The implementation of the augmented system to the linear programming (LP) problem is given in detail in the following:

\section{Equality Constraints}

$$
\begin{gathered}
\sum_{m=1}^{b} \boldsymbol{a}_{m} \boldsymbol{\phi}_{m}+\boldsymbol{r}-\sum_{i=1}^{n} \lambda_{i} \boldsymbol{V}_{i}=\boldsymbol{A}_{1} \quad \boldsymbol{q}^{T}\left(\sum_{m=1}^{b} \boldsymbol{a}_{m} \boldsymbol{\phi}_{m}\right)=1 \\
\boldsymbol{q}^{T}\left(\sum_{i=1}^{n} \lambda_{i} \boldsymbol{V}_{i}\right)=1-\boldsymbol{q}^{T} \boldsymbol{A}_{1}
\end{gathered}
$$

Three basis functions $\phi_{1}, \phi_{2}$, and $\phi_{3}$ with corresponding coefficients $\boldsymbol{a}_{1}, \boldsymbol{a}_{2}$, and $\boldsymbol{a}_{3}$ are used to fully represent the ODFs during a particular process. 


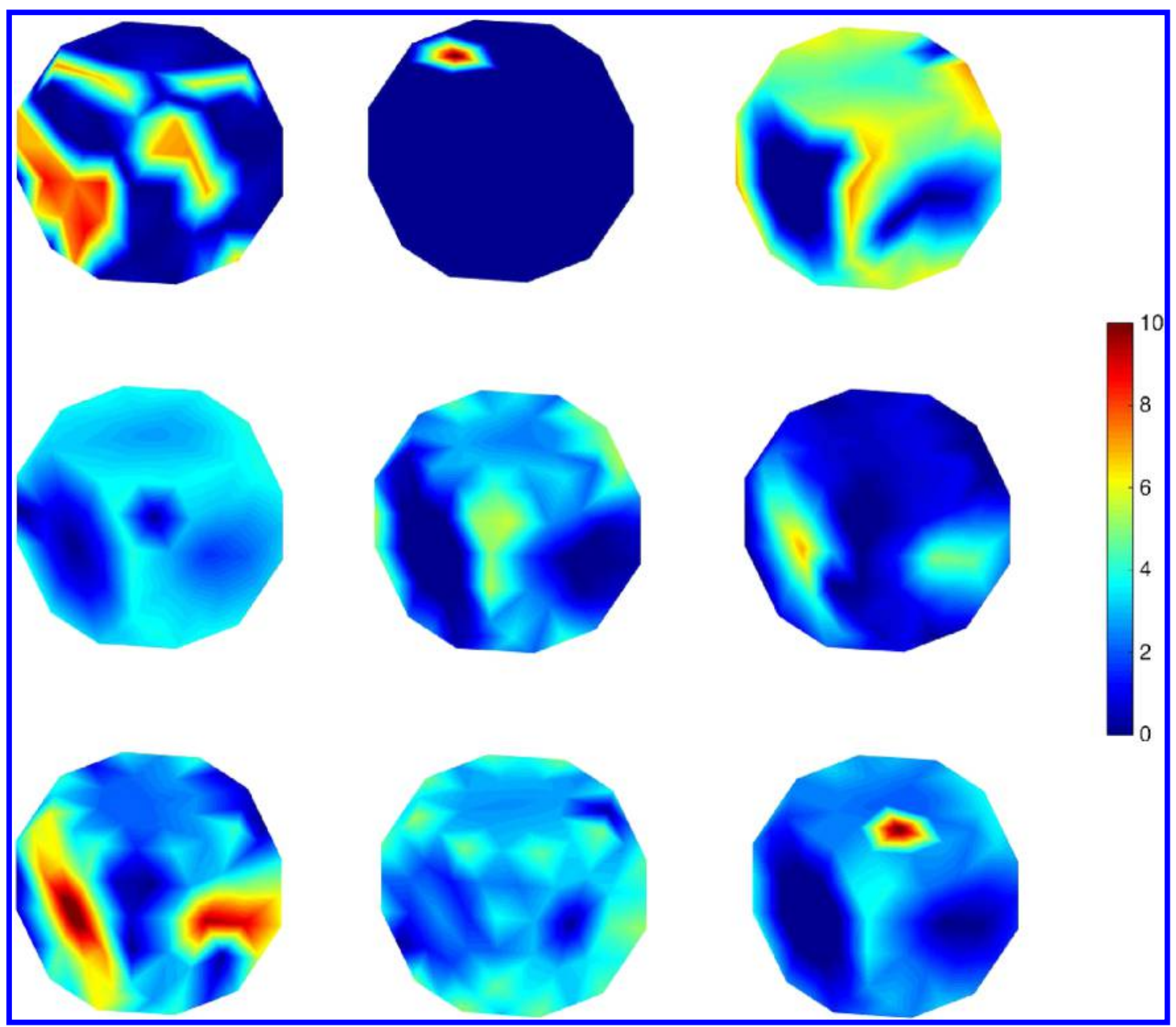

Fig. 6 Optimal ODF examples for galfenol beam microstructure.

2. Augmented System Combining the Equality Constraints

The unknowns in the LP tableau are then written as $\boldsymbol{x}=\left[\boldsymbol{r}_{1}, \ldots, \boldsymbol{r}_{N}, \lambda_{1}, \ldots, \lambda_{n}, \boldsymbol{a}_{1}, \boldsymbol{a}_{2}, \boldsymbol{a}_{3}, \boldsymbol{r}_{0}\right]^{T}$. Thus, the augmented system combining the constraints in Eq. (23) can be written as $\boldsymbol{P}_{\text {aug }} \boldsymbol{x}=\boldsymbol{b}$, where $\boldsymbol{b}=\left[\boldsymbol{A}_{1}^{T}, 1,1-\boldsymbol{q}^{T} \boldsymbol{A}_{1}\right]^{T}$ and

$$
\left[\boldsymbol{P}_{\mathrm{aug}}\right]=\left[\begin{array}{cccccccc}
\boldsymbol{I}_{N \times N} & -\boldsymbol{V}_{1} & \ldots & -\boldsymbol{V}_{n} & \boldsymbol{\phi}_{1} & \boldsymbol{\phi}_{2} & \boldsymbol{\phi}_{3} & 0_{N \times 1} \\
0_{1 \times N} & 0 & \ldots & 0 & \boldsymbol{q}^{T} \boldsymbol{\phi}_{1} & \boldsymbol{q}^{T} \boldsymbol{\phi}_{2} & \boldsymbol{q}^{T} \boldsymbol{\phi}_{3} & 0 \\
0_{1 \times N} & \boldsymbol{q}^{T} \boldsymbol{V}_{1} & \ldots & \boldsymbol{q}^{T} \boldsymbol{V}_{n} & 0 & 0 & 0 & 0
\end{array}\right]
$$

where $\boldsymbol{I}_{N \times N}$ is an $N \times N$ identity matrix. The notation of $0_{N \times 1}$ and $0_{1 \times N}$ indicates the row and column vectors of zeros, respectively.

\section{Inequality Constraints}

Similar augmentations are performed for the inequality constraints for the problem given as

$$
-\boldsymbol{r}_{i}-\boldsymbol{r}_{0} \leq 0 \quad \boldsymbol{r}_{i}-\boldsymbol{r}_{0} \leq 0 \quad \sum_{m=1}^{b} \boldsymbol{a}_{m} \boldsymbol{\phi}_{m} \geq 0 \quad \boldsymbol{A}_{1}+\sum_{i=1}^{n} \lambda_{i} \boldsymbol{V}_{i} \geq 0
$$

\section{Augmented System Combining the Inequality Constraints}

The augmented system combining the constraints in Eq. (24) can be written as $\boldsymbol{M}_{\text {aug }} \boldsymbol{x} \leq \boldsymbol{c}$, where $\boldsymbol{c}=\left[0_{1 \times N}, 0_{1 \times N}, 0_{1 \times N}, \boldsymbol{A}_{1}^{T}\right]^{T}$, and each row of $\boldsymbol{M}_{\text {aug }}$ corresponds to the inequalities in Eq. (24) as indicated in the following:

$$
\left[\boldsymbol{M}_{\mathrm{aug}}\right]=\left[\begin{array}{cccccccc}
-I_{N \times N} & 0 & \ldots & 0 & 0 & 0 & 0 & -1_{N \times 1} \\
I_{N \times N} & 0 & \ldots & 0 & 0 & 0 & 0 & -1_{N \times 1} \\
0_{N \times 1} & 0 & \ldots & 0 & -\boldsymbol{\phi}_{1} & -\boldsymbol{\phi}_{2} & -\boldsymbol{\phi}_{3} & 0 \\
0_{n \times N} & -\boldsymbol{V}_{1} & \ldots & -V_{n} & 0 & 0 & 0 & 0
\end{array}\right]
$$

The notation $1_{N \times 1}$ indicates a vector of ones. The objective is to minimize the bound on the error $\boldsymbol{r}$ given by $\boldsymbol{r}_{0}$. The objective is given as $\boldsymbol{f}^{T} \boldsymbol{x}$, where

$$
\boldsymbol{f}=\left[\begin{array}{llll}
0_{1 \times N} & 0_{1 \times n} & 0_{1 \times 3} & 1
\end{array}\right]^{T}
$$

\section{Final LP Problem}

Thus, the final LP problem reduces to the solution of the following problem:

$$
\min _{a} \boldsymbol{f}^{T} \boldsymbol{x} \text { satisfying the constraints } \quad \boldsymbol{P}_{\mathrm{aug}} \boldsymbol{x}=\boldsymbol{b} \quad \boldsymbol{M}_{\mathrm{aug}} \boldsymbol{x} \leq \boldsymbol{c}
$$

Our objective is to identify the location $\left\{\boldsymbol{a}_{1}, \boldsymbol{a}_{2}, \boldsymbol{a}_{3}\right\}$ on the process plane that best represents the optimal ODF on the material plane computed for the galfenol beam vibration tuning problem. Tension, plane-strain compression and shear are selected as the particular processes, and their basis functions were computed to represent the ODF evolution in these processes. The augmented LP problem is solved for each of these individual processes to identify the closest ODFs on each process plane to the optimal ODFs on the material plane. The values of the objective functions of the LP problems, $\min \boldsymbol{r}_{0}$ and $\max \boldsymbol{\sigma}_{y}$, are compared for each process in Table $\underline{2}$. It 
Table 2 LP problem results for ODF matching on material and process planes

\begin{tabular}{lcc}
\hline \hline Process & $\boldsymbol{r}_{0}$ & $\boldsymbol{\sigma}_{y}, \mathrm{MPa}$ \\
\hline Tension & 0.4689 & 300.0880 \\
Compression & 0.8811 & 296.6571 \\
$x y$ shear & 0.8673 & 298.2191 \\
$x z$ shear & 0.9078 & 301.8826 \\
$y z$ shear & 0.9394 & 299.1096 \\
\hline \hline
\end{tabular}

should be noted that the optimal ODF of the vibration tuning problem provides a yield stress value of $308.4456 \mathrm{MPa}$, and the ODFs on the process plane are expected to provide a close value to the optimum yield stress value.

The results in Table 2 indicate that the ODFs in all the process planes provide similar values of the highest yield strength. The objective, $\min \boldsymbol{r}_{0}$, shows the maximum error value among the independent nodal values in the process and material planes. Thus, the other error values for the rest of the node points in the ODF are less than the objective function value. Since the main objective of this section is to find the best match for the optimal ODF, the best process that provides the closest ODF distribution is the tension process (due to its smallest $\boldsymbol{r}_{0}$ value). The closest microstructure design on the process plane with this approach (approach 1) to the optimum microstructure on the material plane is shown in Fig. 7. The microstructure design in Fig. $\underline{7}$ shows a similar ODF distribution pattern compared to tension process basis functions in Fig. 2. Thus, this microstructure design via a tension deformation process ensures the possibility of manufacturing a very similar ODF to one of the optimum solutions to the galfenol beam vibration tuning problem.

\section{B. Identification of ODFs to Obtain Properties Closest to Desired Set of Properties}

Similar to the optimization problem described in Sec. IV.A where the process plane ODFs closest to optimal ODFs in the material plane were identified, another optimization problem can be posed where the objective is to identify ODFs in the process plane that closely reproduce a desired set of properties. The optimization problem in this case is posed so as to identify ODFs in the process plane for which the properties are closest to a desired set of properties in some sense. This approach is beneficial because the optimum ODFs in the process plane will provide the closest match to the desired property values produced by the optimum ODF solution in the material plane. Even though the problem definition does not provide any control on the ODF values of the material plane, there is still a good possibility of matching the textures in the material and process planes due to matching multiple properties. The objective is to minimize the bound $\left(\boldsymbol{e}_{0} \geq 0\right)$ on the absolute value of error from a desired property:

$$
\min _{a} \boldsymbol{e}_{0}
$$

such that

$$
\sum_{m=1}^{b} \boldsymbol{p}^{T} \boldsymbol{\phi}_{m} \boldsymbol{a}_{m}+\boldsymbol{p}^{T} \boldsymbol{e}=\boldsymbol{d}
$$

where $d$ is the desired set of properties. The normalization constraint is given as

$$
\sum_{m=1}^{b} \boldsymbol{q}^{T} \boldsymbol{\phi}_{m} \boldsymbol{a}_{m}=1
$$

Positivity of the ODF dictates the constraint

$$
\sum_{m=1}^{b} \boldsymbol{\phi}_{m} \boldsymbol{a}_{m} \geq 0
$$

The bound on the absolute value of error is defined as $\left|\boldsymbol{e}_{i}\right| \leq \boldsymbol{e}_{0}$. This is equivalent to pairs of linear inequalities: $-\boldsymbol{e}_{i}-\boldsymbol{e}_{0} \leq 0$ and $\boldsymbol{e}_{i}-\boldsymbol{e}_{0} \leq 0$, where $\boldsymbol{i}=1, \ldots, \boldsymbol{n}_{p}$, and where $n_{p}$ denotes the number of properties to be optimized. This problem uses $\boldsymbol{x}=$ $\left[\boldsymbol{e}_{1}, \ldots, \boldsymbol{e}_{n_{p}}, \boldsymbol{a}_{1}, \ldots, \boldsymbol{a}_{b}, \boldsymbol{e}_{0}\right]^{T}$ as the variable to be identified. The error $\boldsymbol{e}_{i}$ is allowed to be of either sign. The initial desired properties are the design objective (yield stress) and design constraints (natural frequencies) of the galfenol vibration tuning optimization problem. The computation of natural frequencies is not linear, but it is dependent on the stiffness values. Thus, the stiffness parameters, which are calculated with averaging equations, are considered as the representative linear quantities of the design constraints. So, the final desired properties are selected as optimum values of the yield stress and nine independent orthotropic stiffness elements of the vibration tuning problem. The implementation of the augmented system to the LP problem is given in detail in the following:

\section{Equality Constraints}

$$
\boldsymbol{p}^{T}\left(\sum_{m=1}^{b} \boldsymbol{a}_{m} \boldsymbol{\phi}_{m}+\boldsymbol{e}\right)=\boldsymbol{d} \quad \boldsymbol{q}^{T} \sum_{m=1}^{b} \boldsymbol{a}_{m} \boldsymbol{\phi}_{m}=1
$$

Three basis functions $\boldsymbol{\phi}_{1}, \boldsymbol{\phi}_{2}$, and $\boldsymbol{\phi}_{3}$ with corresponding coefficients $\boldsymbol{a}_{1}, \boldsymbol{a}_{2}$, and $\boldsymbol{a}_{3}$ are used to fully represent the ODFs during a particular process.

\section{Augmented System Combining the Equality Constraints}

The unknowns in the LP tableau are then written as $\boldsymbol{x}=$ $\left[\boldsymbol{e}_{1}, \ldots, \boldsymbol{e}_{n_{p}}, \boldsymbol{a}_{1}, \boldsymbol{a}_{2}, \boldsymbol{a}_{3}, \boldsymbol{e}_{0}\right]^{T}$. Thus, the augmented system combining the constraints in Eq. (25) can be written as $\boldsymbol{P}_{\text {aug }} \boldsymbol{x}=\boldsymbol{b}$, where $\boldsymbol{b}=[\boldsymbol{d}, 1]^{T}$ and

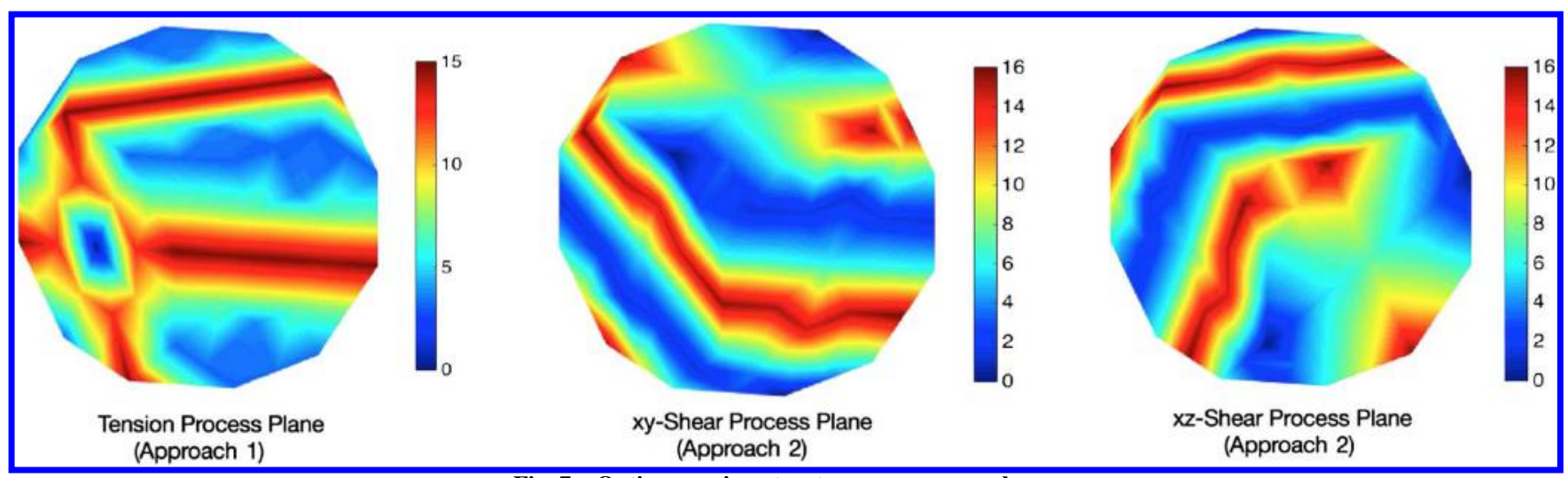




$$
\left[\boldsymbol{P}_{\text {aug }}\right]=\left[\begin{array}{ccccc}
\boldsymbol{p}^{T} & \boldsymbol{p}^{T} \boldsymbol{\phi}_{1} & \boldsymbol{p}^{T} \boldsymbol{\phi}_{2} & \boldsymbol{p}^{T} \boldsymbol{\phi}_{3} & 0 \\
0_{n_{p} \times 1}^{T} & \boldsymbol{q}^{T} \boldsymbol{\phi}_{1} & \boldsymbol{q}^{T} \boldsymbol{\phi}_{2} & \boldsymbol{q}^{T} \boldsymbol{\phi}_{3} & 0
\end{array}\right]
$$

\section{Inequality Constraints}

Similar augmentations are performed for the inequality constraints for the problem given as

$$
-\boldsymbol{e}_{i}-\boldsymbol{e}_{0} \leq 0 \quad \boldsymbol{e}_{i}-\boldsymbol{e}_{0} \leq 0 \quad \sum_{m=1}^{b} \boldsymbol{a}_{m} \phi_{m} \geq 0
$$

\section{Augmented System Combining the Inequality Constraints}

The augmented system combining the constraints in Eq. (26) can be written as $\boldsymbol{M}_{\text {aug }} \boldsymbol{x} \leq 0$, where each row of $\boldsymbol{M}_{\text {aug }}$ corresponds to the inequalities in Eq. (26) as indicated in the following:

$$
\left[\boldsymbol{M}_{\text {aug }}\right]=\left[\begin{array}{ccccc}
-\boldsymbol{I}_{n_{p} \times n_{p}} & 0_{n_{p} \times 1} & 0_{n_{p} \times 1} & 0_{n_{p} \times 1} & -1_{n_{p} \times 1} \\
\boldsymbol{I}_{n_{p} \times n_{p}} & 0_{n_{p} \times 1} & 0_{n_{p} \times 1} & 0_{n_{p} \times 1} & -1_{n_{p} \times 1} \\
0_{n_{p} \times n_{p}} & -\boldsymbol{\phi}_{1} & -\boldsymbol{\phi}_{2} & -\boldsymbol{\phi}_{3} & 0_{n_{p} \times 1}
\end{array}\right]
$$

The objective is to minimize the bound on the error $\boldsymbol{e}$ given by $\boldsymbol{e}_{0}$. The objective is given as $\boldsymbol{f}^{T} \boldsymbol{x}$, where

$$
f=\left[\begin{array}{lll}
0_{1 \times N} & 0_{1 \times 3} & 1
\end{array}\right]^{T}
$$

\section{Final LP Problem}

Thus, the final LP problem reduces to the solution of the following problem:

$$
\min _{a} \boldsymbol{f}^{T} \boldsymbol{x} \text { satisfying the constraints } \quad \boldsymbol{P}_{\text {aug }} \boldsymbol{x}=\boldsymbol{b} \quad \boldsymbol{M}_{\text {aug }} \boldsymbol{x} \leq 0
$$

The problem is to identify the point on a process plane that best represents optimal ODFs in the material plane with desired stiffness properties $\left(C_{11}=281.5559 \mathrm{GPa}, C_{12}=137.3222 \mathrm{GPa}, C_{13}=\right.$ $139.5597 \mathrm{GPa}, \quad C_{22}=296.9677 \mathrm{GPa}, \quad C_{23}=124.1479 \mathrm{GPa}$, $C_{33}=249.7302 \mathrm{GPa}, C_{44}=70.3946 \mathrm{GPa}, C_{55}=85.8063 \mathrm{GPa}$, and $\left.C_{66}=94.2138 \mathrm{GPa}\right)$ and yield stress $\left(\sigma_{y}=308.4456 \mathrm{MPa}\right)$ of the optimum design. The objective function of the LP problem is a measure of the maximum error between the desired property values in the material and process planes. The problem is solved for tension plane-strain compression and shear processes particularly. The results for the objective functions of the LP problems ( $\min \boldsymbol{e}_{0}$ and $\max \boldsymbol{\sigma}_{y}$ ) are shown in Table $\underline{3}$.

The selection of the best processes that represent the closest properties does not depend on the minimum value of $\boldsymbol{e}_{0}$ this time because the sensitivity of various stiffness parameters to the problem objective is different, and all $\boldsymbol{e}_{0}$ values are close to each other. The best process chosen was the one that led to the highest yield stress. According to this criterion, $x z$ shear is the best process for this problem. The ODF designs corresponding to $x y$ - and $x z$-shear processes are shown in Figs. $7 \mathrm{~b}$ and $7 \mathrm{c}$, respectively. The ODF designs that give the closest desired property match on the process plane are not close to the actual ODF solutions in Fig. 6 because the objective of the this problem is to match properties directly rather

Table 3 LP problem results for desired property matching on material and process planes

\begin{tabular}{lcc}
\hline \hline Process & $\boldsymbol{e}_{0}$ & $\boldsymbol{\sigma}_{y}, \mathrm{MPa}$ \\
\hline Tension & 1.9059 & 300.1220 \\
Compression & 1.8681 & 301.1314 \\
$x y$ shear & 1.9578 & 305.6497 \\
$x z$ shear & 1.9338 & 309.0784 \\
$y z$ shear & 1.8343 & 296.7675 \\
\hline \hline
\end{tabular}

Table 4 Reduced-order model coefficients for different methods

\begin{tabular}{lcrrr}
\hline \hline Process & Method & \multicolumn{1}{c}{$a_{1}$} & \multicolumn{1}{c}{$a_{2}$} & \multicolumn{1}{c}{$a_{3}$} \\
\hline Tension & ODF matching & 0.3869 & -0.6403 & -1.6069 \\
Tension & Property matching & 0.3873 & -0.6346 & -1.6065 \\
$x y$ shear & ODF matching & -0.2369 & 0.3195 & -1.5088 \\
$x y$ shear & Property matching & 0.1315 & 0.7459 & -1.4369 \\
$x z$ shear & ODF matching & -0.0878 & 0.4592 & -1.4882 \\
$x z$ shear & Property matching & 0.2173 & -0.5158 & -1.6756 \\
\hline \hline
\end{tabular}

than the optimal ODFs. This also means that none of the processes considered here are able to achieve the global optimum solutions to the problem as identified in Fig. 6. In the future, a sequence of processes (e.g., tension followed by shear) may be investigated instead of using one particular deformation process. To indicate the difference in textures, the reduced-order model coefficients of the optimum deformation processes computed with ODF matching and desired property matching approaches are shown in Table 4 . According to the results in Table 4, every process has a different set of reduced-order model coefficients depending on the solution method. There is especially a considerable difference for $x y$-shear and $x z$-shear processes. Since the basis vectors of any process are always the same, the differences in the reduced-order model coefficients result in different textures for different methods. The ODF matching approach (Sec. IV.A) includes the null-space vectors in the problem definition. Therefore, its ODF values are the closest matches that can be provided by any deformation process. However, the optimum ODF solution in the material plane is not included in the property matching approach. Therefore, each process provides a different set of ODF values for different methods. The difference between the problem definitions of the ODF matching and desired property matching approaches is also the reason that different processes are found to be the optimum solutions of these approaches. According to the results in Secs. IV.A and IV.B, tension is the optimum process to generate the closest texture to the optimum design. However, $x z$ shear is the optimum process to provide the closest property values that are produced by the optimum design.

\section{Conclusions}

This paper develops a microstructure and process optimization methodology for structural problems with a set of macroscale (engineering) design objectives. A two-step approach is followed. In step one, an optimal set of properties is obtained by sampling the property closures, and the multiple orientation distribution functions (ODFs) that lead to these properties are computed using a linear solver. This approach was developed in a previous work. In this paper, an approach is developed to identify microstructures that can be manufactured using a known set of deformation processes. ODFs that can be achieved from various processes are described in a space of reduced-order coefficients (called the process plane), which is obtained using proper orthogonal decomposition. The best processing route is identified by minimizing the distance between any one of the optimal ODFs and the process plane using an augmented linear solver. Another approach where the optimal set of properties is directly projected onto the process plane is also developed. The methods are demonstrated using a vibration tuning problem with the objective of maximizing the yield strength of a galfenol beam. The process plane that is closest to the global optimum ODF is from a tension process. However, the properties of the closest ODF on the process plane are suboptimal. The best set of properties is obtained using an $x z$-shear process on a random (annealed) texture; however, the global optimal ODF solutions identified in step one are not within the set of deformation processes considered here. In the future, a sequence of processes (e.g., tension followed by $x-z$, shear) may be investigated instead of using one particular deformation process to achieve improved properties. 


\section{Acknowledgments}

This work was supported by the U.S. Air Force Office of Scientific Research, U.S. Department of Defense award no. FA9550-12-1-0458 and the National Science Foundation award no. 0954390. The crystal plasticity module was supported by the U.S. Department of Energy under award no. DE-SC0008637.

\section{References}

[1] Ashby, M. F., Materials Selection in Mechanical Design, Pergamon, Tarrytown, NY, 1992, pp. 36-64.

[2] Olson, G. B., "Computational Design of Hierarchically Structured Materials," Science, Vol. 277, No. 5330, 1997, pp. 1237-1242. doi:10.1126/science.277.5330.1237

[3] Sigmund, O., and Torquato, S., "Composites with Extremal Thermal Expansion Coefficients," Applied Physics Letters, Vol. 69, No. 21, 1996, pp. 3203-3205. doi:10.1063/1.117961

[4] Lakes, R., "Deformations in Extreme Matter," Science, Vol. 288, No. 5473, 2000, pp. 1976-1977. doi: $10.1126 /$ science. 288.5473 .1976

[5] Bunge, H. J., Texture Analysis in Materials Science, Butterworths, London, 1982, pp. 3-41.

[6] Kocks, U. F., Tom, C. N., and Wenk, H. R., Texture and Anisotropy, Cambridge Univ. Press, Cambridge, England, U.K., 2000, pp. 44-77.

- [7] Heinz, A., and Neumann, P., "Representation of Orientation and Disorientation Data for Cubic, Hexagonal, Tetragonal and Orthorhombic Crystals," Acta Crystallographica, Vol. A47, No. 6, 1991, pp. 780789. doi:10.1107/S0108767391006864

[8] Adams, B. L., Henrie, A., Henrie, B., Lyon, M., Kalidindi, S. R., and Garmestani, H., "Microstructure-Sensitive Design of a Compliant Beam," Journal of Mechanics and Physics of Solids, Vol. 49, No. 8, 2001, pp. 1639-1663. doi:10.1016/S0022-5096(01)00016-3

[9] Kalidindi, S. R., Houskamp, J., Lyons, M., and Adams, B. L., "Microstructure Sensitive Design of an Orthotropic Plate Subjected to Tensile Load," International Journal of Plasticity, Vol. 20, Nos. 8-9, 2004, pp. 1561-1575.

doi:10.1016/j.ijplas.2003.11.007

[10] Kumar, A., and Dawson, P. R., "Computational Modeling of F.C.C. Deformation Textures over Rodrigues' Space," Acta Materialia, Vol. 48, No. 10, 2000, pp. 2719-2736. doi:10.1016/S1359-6454(00)00044-6

[11] Kumar, A., and Dawson, P. R., "Modeling Crystallographic Texture Evolution with Finite Elements over Neo-Eulerian Orientation Spaces," Computational Methods in Applied Mechanics and Engineering, Vol. 153, Nos. 3-4, 2000, pp. 259-302. doi:10.1016/S0045-7825(97)00072-8
[12] Liu, R., Kumar, A., Chen, Z., Agrawal, A., Sundararaghavan, V., and Choudhary, A., "A Predictive Machine Learning Approach for Microstructure Optimization and Materials Design," Nature Scientific Reports, Vol. 5, 2015, Paper 11551. doi:10.1038/srep11551

[13] Fast, T., Knezevic, M., and Kalidindi, S. R., "Application of Microstructure Sensitive Design to Structural Components Produced from Hexagonal Polycrystalline Metals," Computational Materials Science, Vol. 43, No. 2, 2008, pp. 374-383. doi:10.1016/j.commatsci.2007.12.002

[14] Acar, P., and Sundararaghavan, V., "Utilization of a Linear Solver for Multiscale Design and Optimization of Microstructures," AIAA Journal, Vol. 54, No. 5, 2016, pp. 1751-1759. doi:10.2514/1.J054822

[15] Li, D. S., Garmestani, H., and Adams, B. L., "A Texture Evolution Model in Cubic-Orthotropic Polycrystalline System," International Journal of Plasticity, Vol. 21, No. 8, 2005, pp. 1591-1617. doi:10.1016/j.ijplas.2004.11.009

-116] Sundararaghavan, V., and Zabaras, N., "Linear Analysis of TextureProperty Relationships Using Process-Based Representations of Rodrigues Space," Acta Materialia, Vol. 55, No. 5, 2007, pp. 1573 1587.

doi:10.1016/j.actamat.2006.10.019

[17] Taylor, G. I., "Plastic Strain in Metals," Journal of the Institute of Metals, Vol. 62, 1938, pp. 307-324.

[18] Wenk, H. R., Preferred Orientation in Deformed Metals and Rocks, Academic Press, London, 1985, pp. 73-108.

-[19] Acherjee, S., and Zabaras, N., "A Proper Orthogonal Decomposition Approach to Microstructure Model Reduction in Rodrigues Space with Applications to Optimal Control of Microstructure-Sensitive Properties," Acta Materialia, Vol. 51, No. 18, 2003, pp. 5627-5646. doi:10.1016/S1359-6454(03)00427-0

[20] Ganapathysubramanian, S., and Zabaras, N., "Design Across Length Scales: A Reduced-Order Model of Polycrystal Plasticity for the Control of Microstructure-Sensitive Material Properties," Computer Methods in Applied Mechanics and Engineering, Vol. 193, Nos. 45-47, 2004, pp. 5017-5034.

doi:10.1016/j.cma.2004.04.004

[21] Sundararaghavan, V., and Zabaras, N., "Linear Analysis of Texture Property Relationships Using Process-Based Representations of Rodrigues Space," Acta Materialia, Vol. 55, No. 5, 2007, pp. 1573 1587. doi:10.1016/j.actamat.2006.10.019

[22] Sundararaghavan, V., and Zabaras, N., "On the Synergy Between Texture Classification and Deformation Process Sequence Selection for the Control of Texture-Dependent Properties," Acta Materialia, Vol. 53, No. 4, 2005, pp. 1015-1027. doi:10.1016/j.actamat.2004.11.001

R. Ohayon Associate Editor 
This article has been cited by:

1. Pınar Acar, Siddhartha Srivastava, Veera Sundararaghavan. 2017. Stochastic Design Optimization of Microstructures with Utilization of a Linear Solver. AIAA Journal 55:9, 3161-3168. [Abstract] [Full Text] [PDF] [PDF Plus]

2. Pinar Acar, Siddhartha Srivastava, Veeraraghavan Sundararaghavan. Stochastic Design Optimization of Microstructures with Utilization of a Linear Solver . [Citation] [PDF] [PDF Plus] 